\title{
BMJ Open Effect of triclosan-coated sutures for abdominal wound closure on the incidence of abdominal wound dehiscence: a protocol for an individual participant data meta-analysis
}

\author{
Allard S Timmer (D) , ${ }^{1}$ Niels Wolfhagen (D) , ${ }^{1}$ Frank Pianka, ${ }^{2}$ Phillip Knebel, ${ }^{2}$ \\ Christoph Justinger, ${ }^{3}$ Christos Stravodimos, ${ }^{3}$ Kosuke Ichida (D) , ${ }^{4}$ Toshiki Rikiyama, ${ }^{4}$ \\ József Baracs, ${ }^{5}$ András Vereczkei, ${ }^{5}$ Luca Gianotti, ${ }^{6}$ Jaime Ruiz-Tovar, ${ }^{7}$ \\ Artur M Hernández, ${ }^{8}$ Toru Nakamura, ${ }^{9}$ Marcel G W Dijkgraaf (D),${ }^{10}$ \\ Marja A Boermeester, ${ }^{1}$ Stijn W de Jonge ${ }^{1}$
}

To cite: Timmer AS, Wolfhagen N, Pianka F, et al. Effect of triclosan-coated sutures for abdominal wound closure on the incidence of abdominal wound dehiscence: a protocol for an individual participant data meta-analysis. BMJ Open 2022;12:e054534. doi:10.1136/ bmjopen-2021-054534

- Prepublication history and additional supplemental material for this paper are available online. To view these files, please visit the journal online (http://dx.doi.org/10.1136/ bmjopen-2021-054534)

AST and NW are joint first authors.

MGWD, MAB and SWdJ are joint senior authors.

Received 15 June 2021 Accepted 31 January 2022

\section{Check for updates}

(c) Author(s) (or their employer(s)) 2022. Re-use permitted under CC BY-NC. No commercial re-use. See rights and permissions. Published by BMJ.

For numbered affiliations see end of article.

\section{Correspondence to}

Professor Marja A Boermeester; m.a.boermeester@ amsterdamumc.nl

\section{ABSTRACT}

Introduction Acute abdominal wound dehiscence (AWD) or burst abdomen is a severe complication after abdominal surgery with an incidence up to $3.8 \%$. Surgical site infection (SSI) is the biggest risk factor for the development of AWD. It is strongly suggested that the use of triclosan-coated sutures (TCS) for wound closure reduces the risk of SSI. We hypothesise that the use of TCS for abdominal wound closure may reduce the risk of AWD. Current randomised controlled trials (RCTs) lack power to investigate this. Therefore, the purpose of this individual participant data meta-analysis is to evaluate the effect of TCS for abdominal wound closure on the incidence of AWD.

Methods and analysis We will conduct a systematic review of Medline, Embase and Cochrane Central Register of Controlled Trials for RCTs investigating the effect of TCS compared with non-coated sutures for abdominal wound closure in adult participants scheduled for open abdominal surgery. Two independent reviewers will assess eligible studies for inclusion and methodological quality. Authors of eligible studies will be invited to collaborate and share individual participant data. The primary outcome will be AWD within 30 days after surgery requiring reoperation. Secondary outcomes include SSI, all-cause reoperations, length of hospital stay and all-cause mortality within 30 days after surgery. Data will be analysed with a one-step approach, followed by a two-step approach. In the one-step approach, treatment effects will be estimated as a risk ratio with corresponding $95 \% \mathrm{Cl}$ in a generalised linear mixed model framework with a log link and binomial distribution assumption. The quality of evidence will be judged using the Grading of Recommendations Assessment Development and Evaluation approach.

Ethics and dissemination The medical ethics committee of the Amsterdam UMC, location AMC in the Netherlands waived the necessity for a formal approval of this study, as this research does not fall under the Medical Research involving Human Subjects Act. Collaborating investigators
Strengths and limitations of this study

- Our individual participant data meta-analysis (IPDMA) allows inclusion and analysis of original trial data-including unpublished data on abdominal wound dehiscence (AWD) — and thereby provides detailed information on the effect of triclosan-coated sutures on AWD.

- By this IPDMA, we will be able to check trial data at participant level, standardise inclusion criteria and standardise statistical analysis to minimise heterogeneity, reduce bias and strengthen the conclusion.

- A study limitation is that we aim to collect and analyse trial data of an outcome that was not specified in most of the original studies and individual participant data on this outcome may thus not be available in all trials.

- An IPDMA is statistically challenging and relies on collaboration and input of participating trials.

will deidentify data before sharing. The results will be submitted to a peer-reviewed journal.

PROSPERO registration number CRD42019121173.

\section{INTRODUCTION}

\section{Rationale}

Abdominal wound dehiscence (AWD), also known as acute fascial dehiscence or burst abdomen, is a severe complication after abdominal surgery with a reported incidence of up to $3.8 \% .^{2}$ AWD frequently requires reoperation and is associated with prolonged hospital stay, lower quality of life, increased healthcare costs and mortality rates as high as $45 \% .{ }^{134}$ In the USA, the Nationwide Inpatient Sample demonstrated that AWD results in US\$40323 additional hospital costs per 
patient. ${ }^{5}$ The most important risk factor for the development of AWD is surgical site infection (SSI), increasing the odds more than six times. ${ }^{6}$ Several recently published meta-analyses investigate the effect of the use of triclosancoated suture (TCS) for wound closure; they all report that TCS reduces the risk of SSI. ${ }^{7-10}$ One meta-analysis investigates the effect of TCS on the risk of AWD as a secondary aim, but found that current published trial data provide insufficient information to draw conclusions. ${ }^{11}$ To date, cumulative information of the effect of TCS on the risk of AWD is lacking. Although there are multiple randomised controlled trials (RCTs) investigating the use of TCS for abdominal wound closure, only two describe its effect on the incidence of AWD. ${ }^{12-22}$ The largest trial reports a statistically significant decrease in AWD, but concludes this to be clinically irrelevant as rates of deep SSI are comparable among treatment arms. ${ }^{13}$ Also, the study was not powered to detect a difference in AWD. In the second largest trial, AWD was an exclusion criteria. ${ }^{16}$

An individual participant data meta-analysis (IPDMA) is a meta-analysis of the original trial data and provides the opportunity to include unpublished trial data, standardise inclusion criteria and statistical analysis, check the raw data for integrity and missing data and identify baseline effect modifiers. ${ }^{23}{ }^{24}$ To be able to detect the relative risk that is found in the largest trial (RR 0.42), a study would need 1436 participants. Prior the start of this study, the principle investigators of the two largest trials confirmed that individual participant data (IPD) could be made available. A pooled analysis of just these two trials would contain 2152 participants and therewith easily be able to detect the expected risk difference.

\section{Objectives}

The purpose of this IPDMA is to evaluate the effect of using TCS for abdominal wound closure on the incidence of AWD within 30 days after surgery in patients undergoing open abdominal surgery. Subgroup analyses will be performed according to the specific type of suture that is used for wound closure (polyglactin 910 or polydioxanone) and the level of contamination. We hypothesise that wound closure with TCS reduces the risk of AWD. This may occur through reduction of deep SSI by the use of TCS at the fascial level, or by the use of TCS at more superficial tissue layers reducing superficial SSI and its potential spread to the fascia.

\section{METHODS}

This study consists of a systematic review and a consecutive IPDMA. We will contact authors of studies that meet the inclusion criteria and invite them to contribute to the IPDMA. This study is registered with the International prospective register of systematic reviews (PROSPERO) (registration number CRD42019121173). This protocol is reported according to the Preferred Reporting Items for Systematic Review and Meta-Analysis Protocols (PRISMA-P) statement. ${ }^{25}$ Description and date of all amendments will be reported. The final manuscript will be reported according to PRISMA-Individual Participant Data (PRISMA-IPD) Statement. ${ }^{24}$

\section{Systematic review}

\section{Eligibility criteria}

Randomised trials that investigate the use of TCS, compared with the exact same but non-coated sutures, in patients that underwent open abdominal surgery are potentially eligible. Studies investigating the effect of TCS for abdominal skin closure, and/or abdominal fascia closure will both be eligible. If studies only report the SSI incidence but not the AWD incidence, authors will be asked if AWD incidence is registered (either for the trial or in the medical record for regular care) and available. Trials will only be included if they can share either IPD or aggregated data on the incidence of AWD within 30 days after surgery. If AWD incidence is not available, the study will not be included. We will exclude studies if TCS is part of a bundle of interventions, and studies that investigate the use of TCS after right lower quadrant incision for appendectomy. There will be no restrictions on publication date, language or publication status.

\section{Literature search}

The PubMed (Medline), Embase online databases (Ovid) and Cochrane Central Register of Controlled Trials will be searched. To identify potential unpublished evidence or any ongoing trials, the International Clinical Trials Registry Platform will be searched. References of included studies will be hand searched for any additional relevant studies. In addition, meta-analyses investigating the effect of TCS on the incidence of SSI will be searched for possibly missed eligible studies. The corresponding authors from the collaborating studies will be contacted to review the list of identified studies for omission of potentially relevant studies.

A professional clinical librarian will be consulted to develop the search strategy. The search includes the free text and index terms: sutures, polyglactin 910, vicryl, polydioxanone, PDS, triclosan, wound infection, surgical wound dehiscence, fascial dehiscence and burst abdomen. These terms will be combined with the Cochrane highly sensitive search strategy for identifying randomised trials. ${ }^{26}$ The final search strategy is presented in online supplemental appendix 1.

\section{Study selection}

All studies, identified by the search strategy, will be handled through a free web app ((http://rayyan.qcri. org) ). ${ }^{27}$ Duplicates will be removed. Two reviewers (AST and NW) will independently assess the studies based on previously described eligibility criteria. After screening title and abstract, full text of potentially eligible studies will be retrieved and assessed. When it is not possible to retrieve the manuscript or study eligibility is not clear, the authors will be contacted to provide further information. Any discrepancies in study selection will be resolved 
through discussion or, when necessary, by consultation with the principle investigator. We will keep a list with reasons for exclusions for all articles that pass title and abstract screening but are deemed ineligible for inclusion. Only trials that can provide either IPD or aggregated data on AWD incidence will meet the criteria for final inclusion in the IPDMA.

\section{Individual participant data meta-analysis Study collaboration invitation}

Authors from potentially eligible studies will be contacted and invited to contribute if their study indeed meets the inclusion criteria. An email invitation letter will be sent to the corresponding authors, outlining the IPMDA goals. If no reply is received within 2 weeks, a second email request will be sent to both the corresponding and first author. If again no response is received, we will try to contact all individual authors by email and/or telephone. IPD and/ or aggregated data on AWD will be considered unavailable if numerous times (at least five) no reply is received, if authors no longer have access to the study data or authors do not consent for collaboration. Collaborating investigators will be asked to critically appraise the study protocol, provide feedback, approve the finalised version and will be offered coauthorship on the publication of the study protocol. By sharing their IPD, collaborators will be offered one coauthorship on the IPDMA manuscript, with one additional coauthorship if data of more than 300 participants are shared.

\section{Risk of bias}

Two reviewers (AST and NW) will independently assess the quality of the included studies using the revised tool for assessing Risk of Bias in randomised trials (Rob V.2) ${ }^{28}$ Studies will be judged as 'low risk', 'some concerns' or 'high risk of bias'. Only data from the original manuscripts and study protocols will be used to ensure consistent and uniform assessments of studies that do and studies that do not provide IPD. Presence of publication bias will be assessed with the construction of a contourenhanced funnel plot. $^{29}$

\section{Data collection process}

The collaborating investigators will be requested to sign a data transfer agreement before deidentified IPD is shared. The agreement describes the purposes of the IPDMA, the ownership of the IPD and confirms that the IPD is stored on a secure location. A researcher (AST) will conduct data collection, an interview on the study protocol and a formal handoff of the data codebook, if possible, in person. The primary objective will be to collect IPD for all outcomes. Aggregated data will only be collected if IPD is not available. If aggregated study data are not reported in the publication, this will be requested from the study authors.

\section{Data items}

We will propose a selection of data items of interest (with definitions and measures). All collaborating investigators will be asked to criticise and supplement this list. To ease the process of data handover, collaborating investigators can opt to share the complete data set of their study. We will select and clean only those data items that were selected collaboratively. After repeated consultation with the collaborating investigators, we selected data items on study level and data items on participant level. The list of data items with definitions is presented in online supplemental appendix 2. Study-level data include: study design (number of participating centres, blinding, randomised tissue layer, TCS specification, sample size), inclusion and exclusion criteria, and primary and secondary outcomes. Participant-level data include: baseline characteristics (age, gender, ASA score, body mass index, chronic obstructive pulmonary disease (COPD), smoking status, and previous midline incisions), and procedural characteristics (received suture, procedural status, target organ, wound classification, duration of surgery and incision type).

\section{Outcomes}

The primary outcome is the incidence of AWD requiring reoperation. AWD is defined as spontaneous dehiscence of the abdominal fascia within 30 days postoperatively. Reoperation, for any indication other than AWD, is not regarded as AWD.

Secondary outcomes are incisional SSI within 30 days after surgery according to the Center for Disease Control and Prevention (CDC) criteria (specified as superficial and/or deep), ${ }^{30}$ skin wound dehiscence, length of hospital stay, all-cause reoperations within 30 days after surgery and all-cause mortality within 30 days after surgery.

\section{Data integrity}

IPD will be checked for missing, invalid, out of range and inconsistent outcomes and for discrepancies with the published aggregated data. When detected, we will seek to resolve the issues with the collaborating investigators to improve data quality and ensure that trials are represented accurately. To ensure all randomised participant are included, IPD will be compared with the aggregated data from the original studies. In the case of any concerns on IPD integrity that cannot be resolved with the collaborating investigators, the data of the concerning study will not be included in the primary analysis. Checking baseline imbalances will be used to assess randomisation and allocation concealment. Pattern and extent of follow-up will be checked.

\section{Missing data}

For the primary analysis, we will not perform imputation of the complete variable for a study if variables are systematically missing in one or multiple trials. Missing data at participant level will be assumed to be at random. Multiple imputation by chained equations (MICE) will be used to handle missing data. Multiple rounds of imputation will be used to estimate the missing value. Percentage 
of missing data will determine the number of imputation sets. MICE will be done for each individual trial before merged in the aggregated database.

\section{Data synthesis}

The raw IPD from each study will be copied to a separate database and recoded according to the predefined IPDMA settings. The recoded IPD will then be combined into one database containing the IPD from all studies. Dichotomous data will be expressed using risk ratios (RR) with corresponding 95\% CI. Continuous data will be expressed using weighted mean differences with corresponding 95\% CI. Data will be analysed according to the intention-to-treat principle, meaning that the original randomisation allocation is used to define treatment groups, regardless of the treatment that is actually received.

The primary analysis will be performed in a one-step approach using only IPD. Because the availability of IPD is not an inclusion criterion, it might occur that some trials can only share aggregated data for one or more outcomes. In the additional two-step analysis, aggregated data of outcomes for which IPD are not available will be added and analysed. For the one-step approach, we will use a generalised linear mixed model framework and an appropriate statistical model for the type of outcome. We will use a linear regression model for continuous outcome data and a log-binomial model for binary outcome data. If the log-binomial model fails to converge, we will use a log-binomial generalised estimating equation (GEE) or a $\log$ Poisson GEE model. ${ }^{31}$ A random intercept and, if appropriate, a random slope will be added to account for clustering of patients within studies. Potential confounding variables that, despite randomisation, show baseline imbalances across treatment arms will be added to the appropriate model. Variable selection will be based on VanderWeele ${ }^{32}$ principles of confounder selection. In short, we will control for each variable that is considered a cause of the intervention, the outcome, or both and for any proxy of unmeasured variable that is considered a cause of the intervention and outcome. We will limit the number of variables included in the model by the number of observed events in the dataset with a factor of 1:10. Only variables that are available in all trials are eligible for confounder selection. Additionally, we will perform a twostep approach. In this analysis, IPD from all studies will be reanalysed separately in a similar fashion as the one-step approach but without the term for trial clustering. Aggregated study data of outcomes for which no IPD is available will be added in the two-step approach. The aggregated data of each study will then be summarised, synthesising an overall estimate using DerSimonian and Laird method assuming random effects.

Statistical heterogeneity among studies will be evaluated using the $\chi^{2}$ test and expressed using the $\mathrm{I}^{2}$ statistic. The between-study variance will be assessed using the $\tau^{2}$ statistic. As all tests are prespecified and effects follow from our hypothesis, no correction for multiple testing will be performed.

\section{Additional analyses}

All additional analyses will be performed using the onestep approach. Besides the intention-to-treat analysis, we will perform an as-treated analysis in which participants are analysed according to the type of suture that was actually used rather than the randomisation allocation. When a patient is reoperated, the study-suture is removed and the effect of the used suture on future AWD is diminished if not completely absent. As a result, inclusion of participants that underwent a reoperation might affect the observed treatment effect. We will investigate this in a per-protocol analysis in which participants that underwent a reoperation for any indication other than AWD are excluded. This analysis was added during the peer review process.

Subgroup analyses will be performed according to the specific type of suture that is used for wound closure (polyglactin 910 or polydioxanone), and the level of contamination (according to the CDC criteria).

The risk to develop an incisional hernia is higher after a midline incision than after a non-midline incision. ${ }^{33}$ As such, different incision types may also have different risks for AWD. Inclusion of participant with a non-midline incision introduces some degree of clinical heterogeneity and may affect the observed treatment effect. Therefore, we will perform a sensitivity analysis specifically investigating midline incisions. Additional sensitivity analyses will be performed to assess the effect of the additional use of TCS for skin closure and the effect of adding confounders that pass criteria for confounder selection but are not included in the former model as the variables are not reported in all included studies. Potential bias will further be explored in sensitivity analyses specifically investigating trials that blinded participants and personnel and through exclusion of trials assessed at high risk of bias. A complete case analysis will be performed to investigate the effect of imputation of missing data.

\section{Confidence in cumulative estimate}

The quality of evidence will be judged using the Grading of Recommendations Assessment Development and Evaluation working group methodology for the following domains: risk of bias, unexplained inconsistency, indirectness, imprecision, publication bias, magnitude of effect and residual confounding. ${ }^{34}$ The level of evidence will be downgraded for imprecision based the optimal information size and the CI. If the optimal information size is met and the CI fails to excluded important benefit or harm, we will rate down for imprecision. We set a default threshold for appreciable benefit and harm that warrants rating down (relative risk reduction or RR of $25 \%$ or more). The level of evidence will be upgraded for a large magnitude of effect $(R R>2$ or $<0.5)$ or very large magnitude of effect $(\mathrm{RR}>5$ or $<0.02)$. The overall quality will be 
classified using four levels: high, moderate, low and very low.

\section{Software}

Statistical analysis will be done using R V.4.0.3.

\section{Patient and public involvement}

No patients or patient federations are involved in the design of this study protocol nor the IPDMA. Yet, the disastrous consequences of AWD are well described, underlining the need for (surgical) interventions that reduce the risk of AWD. ${ }^{1}$

\section{Study status}

Currently, we have executed the systematic review. We are in contact with the authors from the original studies. We have not collected any data from the original manuscripts nor received IPD from any of the collaborators.

\section{DISCUSSION}

We designed an IPDMA with the aim to evaluate the effect of using TCS for abdominal wound closure on the incidence of AWD. This protocol describes intended methodology and statistical analysis ahead of analysis to provide transparency and receive timely feedback.

Based on the observed risk difference in the largest published trial, a new RCT investigating the effect TCS on AWD should include around 1500 participants. Such trial would be very time consuming and expose numerous patients to random assignment of two treatments while sufficient information to assess comparative effectiveness may already be available. Moreover, the effect of TCS for wound closure on the risk of SSI is well-documented, and SSI and subsequent AWD risk are closely related. A new RCT is therefore not ethical before the already available information has been optimally analysed.

IPDMA is considered the 'gold standard' in metaanalysis. ${ }^{35}$ At the core of its strength is the use of individual participant data of available trials that allows standardisation of inclusion criteria, definitions and statistical methods to reduce both clinical and statistical heterogeneity. Individual participant data also allow testing of interaction effects to assess subgroup differences and permits exploration of data that was not included in the original publications. Importantly, IPDMA requires intensive collaboration with all trialists on a certain topic, and consequently contributes to consensus on the interpretation of the available data among subject matter experts.

Despite these advantages, an IPDMA has some potential limitations. Its quality depends on the quality, size and number of available studies, the number of included participants, the availability of high-quality data and, most importantly, the willingness to collaborate among the original trialists. We have been incredibly fortunate to find so many of the original researchers willing to collaborate and contribute to the project. The expert input of all involved trialist has greatly contributed to the completion of the study protocol. In consensus meetings, we discussed the differences in data collection and variable definition between the studies. Consequently, we selected a primary outcome for which all studies would be able to uniformly provide data, being AWD requiring reoperation. Despite being a universally available outcome definition, it remains limited by the absence of a strict criteria on when to reoperate. Variation between clinicians exists and the consideration on whether or not to reoperate is hard if not impossible to retrieve. As selective reoperation by biased investigators may affect the results, we will perform a sensitivity analysis only including trials that blinded both participants and personnel making selective reoperation near impossible. Blinding for allocation is easily performed because the sutures look identical.

In conclusion, this study protocol describes an individual participant data meta-analysis in which we aim to investigate if the use of TCS for abdominal wound closure reduces the risk of AWD. If a lower incidence of AWD is observed, this may have considerable consequences for daily practice.

\section{ETHICS AND DISSEMINATION \\ Ethical approval}

All individual trials were approved by a medical ethics committee according to national legislation. The medical ethics committee of the Amsterdam UMC, location AMC in the Netherlands waived the necessity for a formal approval of this study, as this research does not fall under the Medical Research involving Human Subjects Act.

\section{Dissemination}

The results of this study will be submitted to peer-reviewed journals regardless of the outcome. The protocol will be submitted before the data are gathered and analysed.

\section{Author affiliations}

${ }^{1}$ Department of Surgery, Amsterdam Gastroenterology and Metabolism, Amsterdam Infection and Immunity, Amsterdam UMC, Location AMC, Amsterdam, The Netherlands

${ }^{2}$ Department of General, Visceral and Transplantation Surgery, UniversitatsKlinikum Heidelberg, Heidelberg, Germany

${ }^{3}$ Department of Surgery, Städtisches Klinikum Karlsruhe gGmbH, Karlsruhe, Germany

${ }^{4}$ Department of Surgery, Jichi Medical University, Saitama, Japan

${ }^{5}$ Department of Surgery, University of Pecs, Pecs, Hungary

${ }^{6}$ Department of Surgery, San Gerardo Hospital, Monza, Italy

${ }^{7}$ Department of Surgery, Rey Juan Carlos University, Madrid, Spain

${ }^{8}$ Department of Humanities and Social Sciences, University Isabel I, Burgos, Spain

${ }^{9}$ Department of Surgery, Hokkaido University, Sapporo, Japan

${ }^{10}$ Department of Epidemiology and Data Science, Amsterdam UMC, Amsterdam

Public Health, University of Amsterdam, Amsterdam, The Netherlands

Acknowledgements The authors would like to thanks F.S. van Etten - Jamaludin, clinical librarian for the help with the literature search.

Contributors MAB is guarantor of the study. SWdJ and MAB conceived the study. AST, NW, SWdJ, MGWD and MAB designed the study, drafted the study protocol and provided statistical expertise. AST and NW provided input for the literature search and will coordinate the assembly of the data and perform the screening, inclusion and assessment of risk of bias and they also contributed equally to this paper. $F P$, PK, CJ, CS, KI, TR, JB, AV, LG, JR-T, AMH and TN provided substantial contributions to the study design, provided critical feedback and approved the final version of the study protocol. All authors compliant with their responsibilities according to the research protocol, meet authorship criteria as defined by the international committee of medical journal editors. 
Funding This study is supported by a grant from Johnson \& Johnson.

Disclaimer This is an investigator-initiated study. The sponsor of this study is the Amsterdam UMC, location Amsterdam, the Netherlands. The study protocol is written solely by members of the steering committee and the industry had no say in study design, data items, data collection, data analysis and data reporting.

Competing interests AST, NW, FP, PK, CS, Kl, TR, JB, AV, LG, JR-T, AMH, TN, MGWD and SWdJ declare no conflict of interest. $\mathrm{CJ}$ is an advisory board member of Johnson \& Johnson/Ethicon. MAB reports receiving institutional grants from J\&J/ Ethicon, $\mathrm{KCl} / 3 \mathrm{M}$, and New Compliance; and is an advisory board member and/or speaker and/or instructor for KCl/3M, Johnson \& Johnson/Ethicon, LifeCell/Allergan, Bard, Gore, TelaBio, Medtronic, GD Medical, and Smith \& Nephew.

\section{Patient consent for publication Not applicable.}

Provenance and peer review Not commissioned; externally peer reviewed.

Supplemental material This content has been supplied by the author(s). It has not been vetted by BMJ Publishing Group Limited (BMJ) and may not have been peer-reviewed. Any opinions or recommendations discussed are solely those of the author(s) and are not endorsed by BMJ. BMJ disclaims all liability and responsibility arising from any reliance placed on the content. Where the content includes any translated material, BMJ does not warrant the accuracy and reliability of the translations (including but not limited to local regulations, clinical guidelines, terminology, drug names and drug dosages), and is not responsible for any error and/or omissions arising from translation and adaptation or otherwise.

Open access This is an open access article distributed in accordance with the Creative Commons Attribution Non Commercial (CC BY-NC 4.0) license, which permits others to distribute, remix, adapt, build upon this work non-commercially, and license their derivative works on different terms, provided the original work is properly cited, appropriate credit is given, any changes made indicated, and the use is non-commercial. See: http://creativecommons.org/licenses/by-nc/4.0/.

\section{ORCID iDs}

Allard S Timmer http://orcid.org/0000-0002-9990-7192

Niels Wolfhagen http://orcid.org/0000-0003-0573-5703

Kosuke Ichida http://orcid.org/0000-0002-0533-8698

Marcel G W Dijkgraaf http://orcid.org/0000-0003-0750-8790

\section{REFERENCES}

1 van Ramshorst $\mathrm{GH}$, Eker $\mathrm{HH}$, van der Voet JA, et al. Long-term outcome study in patients with abdominal wound dehiscence: a comparative study on quality of life, body image, and incisional hernia. J Gastrointest Surg 2013;17:1477-84.

2 Mazilu O, Grigoraș D, Cnejevici S, et al. [Postoperative complete abdominal dehiscence: risk factors and clinical correlations]. Chirurgia 2009;104:419-23.

3 Carlson MA. Acute wound failure. Surg Clin North Am 1997;77:607-36.

4 Fleischer GM, Rennert A, Rühmer M. [Infected abdominal wall and burst abdomen]. Chirurg 2000;71:754-62.

5 Shanmugam VK, Fernandez SJ, Evans KK, et al. Postoperative wound dehiscence: predictors and associations. Wound Repair Regen 2015;23:184-90.

6 van Ramshorst GH, Nieuwenhuizen J, Hop WCJ, et al. Abdominal wound dehiscence in adults: development and validation of a risk model. World J Surg 2010;34:20-7.

7 Ahmed I, Boulton AJ, Rizvi S, et al. The use of triclosan-coated sutures to prevent surgical site infections: a systematic review and meta-analysis of the literature. BMJ Open 2019;9:e029727.

8 de Jonge SW, Atema JJ, Solomkin JS, et al. Meta-analysis and trial sequential analysis of triclosan-coated sutures for the prevention of surgical-site infection. Br J Surg 2017;104:e118-33.

9 Henriksen NA, Deerenberg EB, Venclauskas L, et al. Triclosancoated sutures and surgical site infection in abdominal surgery: the TRISTAN review, meta-analysis and trial sequential analysis. Hernia 2017;21:833-41.

10 Uchino M, Mizuguchi T, Ohge H, et al. The efficacy of AntimicrobialCoated sutures for preventing incisional surgical site infections in digestive surgery: a systematic review and meta-analysis. $J$ Gastrointest Surg 2018;22:1832-41.

11 Elsolh B, Zhang L, Patel SV. The effect of Antibiotic-Coated sutures on the incidence of surgical site infections in abdominal closures: a meta-analysis. J Gastrointest Surg 2017;21:896-903.
12 Baracs J, Huszár O, Sajjadi SG, et al. Surgical site infections after abdominal closure in colorectal surgery using triclosan-coated absorbable suture (PDS plus) vs. uncoated sutures (PDS II): a randomized multicenter study. Surg Infect 2011;12:483-9.

13 Diener MK, Knebel P, Kieser M, et al. Effectiveness of triclosancoated PDS plus versus uncoated PDS II sutures for prevention of surgical site infection after abdominal wall closure: the randomised controlled PROUD trial. Lancet 2014;384:142-52.

14 Galal I, El-Hindawy K. Impact of using triclosan-antibacterial sutures on incidence of surgical site infection. Am J Surg 2011;202:133-8.

15 Ichida K, Noda H, Kikugawa R, et al. Effect of triclosan-coated sutures on the incidence of surgical site infection after abdominal wall closure in gastroenterological surgery: a double-blind, randomized controlled trial in a single center. Surgery 2018. doi:10.1016/j.surg.2017.12.020. [Epub ahead of print: 10 Mar 2018].

16 Justinger C, Slotta JE, Ningel S, et al. Surgical-site infection after abdominal wall closure with triclosan-impregnated polydioxanone sutures: results of a randomized clinical pathway facilitated trial (NCT00998907). Surgery 2013;154:589-95.

17 Mattavelli I, Rebora P, Doglietto G, et al. Multi-Center randomized controlled trial on the effect of triclosan-coated sutures on surgical site infection after colorectal surgery. Surg Infect 2015;16:226-35.

18 Nakamura T, Kashimura N, Noji T, et al. Triclosan-coated sutures reduce the incidence of wound infections and the costs after colorectal surgery: a randomized controlled trial. Surgery 2013;153:576-83.

19 Olmez T, Berkesoglu M, Turkmenoglu O, et al. Effect of triclosancoated suture on surgical site infection of abdominal fascial closures. Surg Infect 2019;20:658-64.

20 Rasić Z, Schwarz D, Adam VN, et al. Efficacy of antimicrobial triclosan-coated polyglactin 910 (Vicryl* plus) suture for closure of the abdominal wall after colorectal surgery. Coll Antropol 2011;35:439-43.

21 Ruiz-Tovar J, Alonso N, Morales V, et al. Association between triclosan-coated sutures for abdominal wall closure and incisional surgical site infection after open surgery in patients presenting with fecal peritonitis: a randomized clinical trial. Surg Infect 2015;16:588-94.

22 Ruiz-Tovar J, Llavero C, Jimenez-Fuertes M, et al. Incisional surgical site infection after abdominal fascial closure with triclosan-coated barbed suture vs triclosan-coated polydioxanone loop suture vs polydioxanone loop suture in emergent abdominal surgery: a randomized clinical trial. J Am Coll Surg 2020;230:766-74.

23 Riley RD, Lambert PC, Staessen JA, et al. Meta-analysis of continuous outcomes combining individual patient data and aggregate data. Stat Med 2008;27:1870-93.

24 Stewart LA, Clarke M, Rovers M, et al. Preferred reporting items for systematic review and meta-analyses of individual participant data: the PRISMA-IPD statement. JAMA 2015;313:1657-65.

25 Moher D, Shamseer L, Clarke M, et al. Preferred reporting items for systematic review and meta-analysis protocols (PRISMA-P) 2015 statement. Syst Rev 2015;4:1.

26 Higgins JP, Se G. Cochrane Handbook for Systematic Reviews of Interventions Version 5.1.0 [updated March 2011], 2011. The Cochrane Collaboration. Available: www.handbook.cochrane.org

27 Ouzzani M, Hammady H, Fedorowicz Z, et al. Rayyan-a web and mobile app for systematic reviews. Syst Rev 2016;5:210.

28 Sterne JAC, Savović J, Page MJ, et al. RoB 2: a revised tool for assessing risk of bias in randomised trials. BMJ 2019;366:14898.

29 Peters JL, Sutton AJ, Jones DR, et al. Contour-enhanced metaanalysis funnel plots help distinguish publication bias from other causes of asymmetry. J Clin Epidemiol 2008;61:991-6.

30 Mangram AJ, Horan TC, Pearson ML, et al. Guideline for prevention of surgical site infection, 1999. Hospital infection control practices Advisory Committee. Infect Control Hosp Epidemiol 1999;20:250-78. quiz 79-80.

31 Pedroza C, Truong VTT. Estimating relative risks in multicenter studies with a small number of centers - which methods to use? A simulation study. Trials 2017;18:512.

32 VanderWeele TJ. Principles of confounder selection. Eur J Epidemiol 2019;34:211-9.

33 Halm JA, Lip H, Schmitz PI, et al. Incisional hernia after upper abdominal surgery: a randomised controlled trial of midline versus transverse incision. Hernia 2009;13:275-80.

34 Guyatt GH, Oxman AD, Vist GE, et al. GRADE: an emerging consensus on rating quality of evidence and strength of recommendations. BMJ 2008;336:924-6.

35 Riley RD, Lambert PC, Abo-Zaid G. Meta-analysis of individual participant data: rationale, conduct, and reporting. BMJ 2010;340:c221. 